\title{
Urethral Catheterization: A Review of the Indications, Techniques, and Complications of Male Urethral Catheterization for General Medical Practice
}

\author{
Akanmode $\mathrm{AM}^{1}$, Ekabua $\mathrm{JJ}^{2}$, Eketunde $\mathrm{AO}^{3 *}$, Osanoto $\mathrm{AS}^{4}$, Acholonu $\mathrm{CU}^{1}$ and Origbemisoye $\mathrm{AM}^{5}$ \\ ${ }^{1}$ Clarendon Health Department, Ministry of Health \& Wellness, Jamaica \\ ${ }^{2}$ Windsor University School of Medicine, Caribbean \\ ${ }^{3}$ Public Health, University of Massachusetts, Lowell, USA \\ ${ }^{4}$ Department of Human Anatomy and Embryology, University of Health Sciences, Antigua and Barbuda \\ ${ }^{5}$ Department of General Surgery, May Pen General Hospital, Jamaica
}

"Correspondence: Adenike O Eketunde, Public Health, University of Massachusetts, Lowell, USA

Received on 20 August 2020; Accepted on 17 September 2020; Published on 24 September 2020

Copyright (C) 2020 Akanmode A. This is an open access article and is distributed under the Creative Commons Attribution License, which permits unrestricted use, distribution, and reproduction in any medium, provided the original work is properly cited.

\begin{abstract}
The physiologic process of micturition plays an essential role in the ability of the human body to regulate homeostasis. When the urinary system encounters an obstruction such as a foreign body within the bladder or other prostatic diseases like benign prostatic hyperplasia (BPH), alternative measures to drain the bladder is required, this birthed the use of urethral catheters $\&$ the catheterization procedure. Urethral catheterization dates to the early days of medicine and while it is mostly a routine procedure in this era, the total understanding of its indications, proper techniques, and associated complications remains an essential tool in the arsenal of a practicing physician.
\end{abstract}

Keywords: catheterization, catheters, bladder, urinary retention

Abbreviations: BPH: benign prostatic hyperplasia; CBI: continuous bladder irrigation

\section{Brief History of Catheters}

"Kathiénai," which translates as "to thrust into," was used by the Greeks to describe catheters. The history of bladder catheterization extends way back to $1500 \mathrm{BC}$, as recorded by an early Egyptian papyrus, transurethral bronze tubes, straws \& reeds were used to relieve urinary retention. Al-Zahrawi (Albucasis), the great Arab-Andalusian surgeon also described the use of flexible silver tubes with multiple side holes for more comfortable transurethral relief of 
urinary retention. Other early civilizations, such as the Chinese, also fashioned various transurethral devices to relieve urinary retention [1].

The famous inventor Benjamin Franklin (1706-1790) devised a helical silver wired tube rubbed with fat for his brother. The latter had urinary retention due to bladder stones.

In the 18th century, catheters made from rubber were developed, but this had a drawback initially as such catheters were weak at body temperatures and were notorious for leaving rubber debris within the bladder. With the advent of rubber vulcanization technique by Charles Goodyear in 1844, rubber catheters became more durable and resilient [2].

Dr Frederic Foley introduced a latex balloon catheter, which is currently known as Foley catheter has undergone various modifications for modern-day medical use. At the same time, Charles French, a Parisian surgical instrument maker, devised the French (Fr) scale used to illustrate the external diameter of a catheter [3].

\section{Urethral Catheters}

The dwell time concept applies to urethral catheter use. Catheters can be inserted into the bladder for a short duration, such as an in and out immediate drainage of urinary retention or attached to a collection device for urinary output measurements during surgical procedures. Catheters can also be inserted for a longer duration or left indwelling when dealing with patients with chronic urinary retention [4].

There are three major types of urinary catheters:

- External catheters: this is also known as condom catheters, and as the name suggests, they are placed over the penis like a condom, they are generally more comfortable.

- Internal catheters: this can either be a transurethral or suprapubic catheter; these categories of catheters are also referred to as indwelling catheters.

- Short term catheters: they are also referred to as intermittent catheters.

\section{Catheter Parameters}

- Catheter diameters: catheter sizes are derived from the French (Fr) scale and $1 \mathrm{Fr}=0.33 \mathrm{~mm}$ thus, an 18 Fr catheter is $6 \mathrm{~mm}$ in diameter.

- Catheter length: the length of a catheter tends to vary depending on the length of the urethra. A typical male catheter is $40-45 \mathrm{~cm}$ long; a female catheter is $20-26 \mathrm{~cm}$ long while a pediatric catheter is $30-31 \mathrm{~cm}$ long.

- Catheter materials: catheters are made of different materials such as:

* Latex: latex catheter can elicit an anaphylactic reaction in latex allergic patients. Thus, they are generally used for short term catheterization purposes.

* Silicone: $100 \%$ silicone catheters can be used for a longer duration of about 12 weeks, and they are hypoallergenic.

* Silicone elastomers: these categories of catheters are made of a latex core coated with silicone; they are more flexible and can have a longer dwell time, but still not favorable for latex allergic patients.

* Plastics/PVC's: catheters made from these materials tend to be stiff and uncomfortable for the patients, thus their use is limited to a short duration.

* Catheter balloon: the balloon of the catheter is synonymous with the anchor on a ship. It must be handled carefully by inflating it to the appropriate diameter as recommended by the manufacturer. Catheter balloon has different sizes:

- The pediatric catheter is inflated to $5 \mathrm{ml}$.

- $10 \mathrm{ml}$ for standard use catheters.

- $30 \mathrm{ml}$ for post-operative use. 
It is best to inflate the balloon with sterile water or, as per the manufacturer's recommendation, the use of air, regular water, and saline is not appropriate. Saline can crystallize in the inflating balloon or within the channels, thus posing a problem when trying to retrieve the catheter [5].

\section{Relevant Anatomy and Physiology}

The urinary system in humans comprises of a pair of kidneys, the ureters, the vesicular bladder, and the urethra. For this article, the focus is on the bladder and the urethra.

\section{Bladder Anatomy}

The human bladder is in the extraperitoneal area, and it lies posterior to the pubic symphysis. It is a muscular and distensible organ whose shape is varying based on its content and those of the surrounding organs. The bladder is divided into four major parts [6]:

- The apex: this points to the superior aspect of the pubic symphysis.

- The fundus: this is the portion of the bladder opposite the apex.

- The body: this is the portion of the bladder located between the apex and the body.

- Bladder neck: this is formed by the convergence of the inferolateral aspects and the fundus.

The bladder wall is structurally made up of 3 components, namely:

- The outer layer: is made up of loose connective tissues and blood vessels.

- The middle layer: this is the muscular layer of the bladder. The musculature of the bladder is a unique one; it is made of detrusor muscle fibers oriented in multiple directions; this helps retain the bladder's structural integrity when it is stretched. The detrusor fibers receive both sympathetic and parasympathetic innervations. The internal urethral sphincter, which contracts during ejaculation to prevent a retrograde ejaculation, is also made up of the detrusor muscle fibers [7].

- The inner layer: this is made up mostly of transitional type epithelium.

\section{Bladder Physiology}

The bladder structurally acts as a storage location for urine until the central nervous system initiates the micturition reflex. The bladder capacity is one that relatively changes with increasing age. The approximate bladder volume for children can be calculated with the formula (years of age +2$) \times 30 \mathrm{ml}$.

In adults, the average bladder can hold between 300-400 $\mathrm{ml}$ of urine. With increasing bladder volume, there is increased pressure on the walls of the bladder. The wall pressure range of 5-15 $\mathrm{mmHg}$ elicits bladder fullness sensations, which is transmitted to the spinal cord to stimulate the micturition reflex [8].

\section{Urethral Anatomy and Physiology}

The urethra is a long hollow muscular tube that serves to convey urine and other waste from the vesical bladder to the external urethral orifice, which is located at the tip of the glans penis. The urethral also serves to convey semen produced during ejaculation similarly. The urethral is approximately $18-22 \mathrm{~cm}$ long in males and about $4 \mathrm{~cm}$ long in females. For this purpose of this article, our focus is on the male urethra anatomy and physiology.

The urethra is composed of 3 significant segments:

- The proximal prostatic urethra: this segment of the urethra is within the prostate, and it is the most common part of the prostate affected by benign prostatic hyperplasia (BPH).

- Membranous urethra: this is the shortest and the most rigid part of the urethra. Due to its anatomy, this part of the urethral is the most susceptible to urethral injuries arising from pelvic traumas. 
- Spongy urethra: this segment of the urethra traverses the spongy corpus callosum of the penis hence its name; it is further divided into two parts: the pendulous urethra and the bulbous urethral [9].

Excellent knowledge of the urethral and bladder anatomy is essential for a successful bladder catheterization procedure.

\section{Indications for Bladder Catheterization}

The evidence for bladder catheterization can be broadly sub-divided into therapeutic and diagnostic indications [3, 5, 10].

Therapeutic indications for urethral catheterization:

- Acute urinary retention relief: acute urinary retention is usually seen in urgent care and emergency department, but occasionally a good number of cases are recognized by the general practitioner. Most cases of acute retention are caused by BPH or blood clots.

- For initiating continuous bladder irrigation (CBI): bladder catheterization via a three-way Foley catheter is utilized for CBI, a long-standing procedure used in the management of gross hematuria and other bladder problems.

- Intermittent decompression of neurogenic bladder: when bladder detrusor muscles malfunction due to neurogenic injury from traumas and diseases, urethral catheterization is warranted. Patients with neurogenic bladder benefit significantly from intermittent bladder catheterization; this is particularly beneficial to younger patients with neurogenic bladder arising from spinal cord injury instead of indwelling catheters.

- Immobilization: immobilized patients due to pelvic or lumbar fractures require bladder catheterization to aid with voiding urine.

- Hygienic management of bedridden patients: a short-term or long-term indwelling catheter can be utilized when managing the sanitary needs of elderly or bedridden patients.

- Intravesical administration of medications: bladder catheterization via the urethra helps with administering specific pharmacologic agents such as the BCG vaccine and other chemotherapy agents when managing urothelial carcinoma and superficial bladder cancers [11, 12].

Diagnostic indications for urethral catheterization:

- Collection of catheter urine specimen: catheter specimen urine (CSU) aids with laboratory analysis of urine samples. This is particularly important when contamination of the urine sample is plausible.

- Urine output monitoring: an accurate monitoring of urine output is essential in assessing kidney function, especially in an operating room setting. The use of specific pharmacologic agents such as diuretics when managing fluid overload patients due to cardio-renal compromise also requires bladder catheterization for accurate input/output fluid monitoring.

- Urinary tract imaging: The use of specific contrast dye agent is required during imaging of the urinary system. Bladder catheterization via the urethra helps with the introduction of the dye [13].

\section{Contraindications to Catheterization}

Urethral injury: the only absolute contraindication to the insertion of a urinary catheter in males is the presence of a traumatic injury to the lower urinary tract, such as a urethral tear.

History of saddle type accident or pelvic injuries during motor vehicle accidents or other causes coupled with examination findings of a high riding or a boggy prostate, hematoma at the perineum, or the classic presence of blood at the urethral meatus should warrant a high index of suspicion for a urethral injury.

A retrograde urogram is usually performed when any suspicion of urethral injury is raised [14]. 


\section{Equipment}

There are various types of equipment used to facilitate a successful urethral catheterization, while most facilities have a single-use commercial catheterization set, others don't, and the individual pieces of instruments listed below can also be assembled for catheterization.

- $10 \%$ povidone-iodine solution: catheterization is a sterile procedure; adequate cleaning of the insertion site is optimal to avoid the introduction of disease-causing microbes into the urinary tract.

- Urethral catheters: the catheters come in various shapes, lengths, and diameters; the French (Fr) scale indicates the size of a catheter. Larger catheters are usually used for males, as they are stiffer and can easily traverse the longer urethra of males.

* Adult male: the (16-18 Fr) straight tip Foley catheter is mostly recommended during urethral catheterization.

- Adult male with prostrate obstruction: obstruction at the level of the prostrate is primarily due to $\mathrm{BPH}$, the use of coude tip $18 \mathrm{Fr}$ catheter is beneficial in this cohort of patients as it can easily bypass the prostatic blockage and efficiently drain the urinary bladder alternatively a large 2-way Foley catheter (20-24 Fr) can be utilized.

* Males with hematuria: a 3-way catheter (20-30 Fr) is used during hematuria to commence CBI.

* Male children and infants: for an accurate estimation of the size of a urinary catheter in children (child's age $\div 2$, then add 8 ) while in the infant using the feeding tube (5 Fr) is utilized.

- Sterile gloves

- Cotton balls.

- A water-soluble lubricating gel or anesthetic gel: an appropriate water-soluble gel ensures easy insertion of the urinary catheter [9].

- 10cc syringe: the syringe is prefilled with $0.9 \%$ normal saline solution. A calibrated urine collection bag [15].

- Sterile drapes.

\section{Personnel}

Urethral catheterization is a relatively simple procedure; minimal help is required by the procedural assistant to ensure a successful procedure.

\section{Preparation}

- Informed consent: like many other routine procedures, patients evaluated and found to fit the criteria for urethral catheterization should have the process explained to them in detail and should have a firm understanding of the benefits and risks involved. When this is done, written consent must be documented on the patient's clinical records.

- Positioning: the ideal position for urethral catheterization is that of the 'frog legged position'. Here the knees are flexed, and the hips are abducted to a $45^{\circ}$ angle.

- Equipment preparation: the clinician should wash his hand properly first to clean and then ensure that the trolley and the plastic tray is cleaned with an aseptic solution like chlor-clean. Once this is achieved, the single-use catheter insertion package or the individual's pieces of equipment can be gathered on the tray. When a single-use catheterization set is available same should be opened in a sterile manner. The iodine solution applied to the cotton balls, and the lidocaine gel packet cut open.

- Patient exposure: ensure that the patient is adequately exposed from the waist down and their dignity appropriately maintained [10]. 


\section{Technique}

- Don the sterile gloves in a proper method.

- Make a hole in the center area of the sterile drapes and place it over the penis forming a sterile field.

- With the non-dominant hand to hold the penis firmly but not too tight and gently retract the prepuce if uncircumcised with the fingers.

- The sterile dominant hand holds the forceps and picks up the cotton soaked in $10 \%$ povidone-iodine solution and cleans the glans penis and penile shaft from the tip to the base.

- Use the $10 \mathrm{ml}$ syringe with no needle attached to gently install 5-10 $\mathrm{ml}$ of $2 \%$ lidocaine gel into the urethra and allow a few minutes to let the anesthetic agent take effect before inserting the catheter. To prevent leakage of the lidocaine gel, the clinician can either gently squeeze the tip of the penis or place a finger over the external urethral opening [16].

- Carefully open the catheter from its sterile pack exposing the tip only.

- Without touching the tip of the catheter, insert it slowly and gently into the urethra of the penis held at $90^{\circ}$ until the Y-shaped portion of the catheter meets the urethral meatus.

- Wait for the urine return to ensure that the distal end of the catheter is within the bladder. Once urine flow is observed from the larger port of the catheter, inflate the catheter's balloon with the right amount of sterile water as per the catheter manufacturer's specification.

- Tug the catheter gently to ensure that the balloon well anchors it against the bladder wall.

- Attach the urinary bag and let the urine drain.

- Replace the foreskin of the penis in uncircumcised patients to prevent paraphimosis.

- Clean the patients surrounding and discard all waste into the appropriate biohazard bin.

- Remove your gloves and decontaminate your hands [8,10,17].

\section{Special Scenarios}

- No urine flow: with the successful advancement of the catheter as described above, if no urinary return is noted immediately, the clinician should exercise some patience as the lubricating gel occasionally occludes the tip of the catheter. If no spontaneous urine discharge is observed, a $60 \mathrm{ml}$ syringe can be attached to try and aspirate urine. If no urine is still noted after this procedure, the clinician should withdraw the catheter and repass it again (this is usually done after confirming the presence of urine within the vesical bladder with ultrasonography) $[10,15]$.

- Coude catheter insertion: coude catheters are generally stiffer and have a curved tip designed to overcome the obstruction, especially for patients with BPH. To pass the coude catheter, the procedure described above should be followed. The tip of the catheter must face anteriorly to ensure that the rounded ball at the tip of the catheter navigates through the urogenital diaphragm.

- Suprapubic catheterization: suprapubic catheterization shares many of the techniques as urethral catheterization. When urethral catheterization is contraindicated or unsuccessful, a suprapubic catheter is inserted by making an incision above the pubic symphysis along the midline. The catheter is passed into the bladder. The decision to perform suprapubic catheterization is usually restricted to the urologist or the general surgeon. At the same time, subsequent catheter management can be undertaken at an outpatient setting by the general care provider $[18,19]$.

- Leakage: for patients that require a long duration of catheterization, it is not uncommon for them to have a spasm of the bladder, this spasm creates a leakage around the catheter. When such leakages are encountered, a larger Foley catheter should be utilized to correct the same. When bladder spasm is due to detrusor muscle instability, antispasmodic agents such as oxybutynin can be used [15]. 


\section{Removal of Urethral Catheter}

Removing a urethral catheter is a relatively simple procedure; with the aid of an empty syringe, the catheter balloon is deflated, and the catheter gradually retracted. If significant pain and discomfort catheter balloon is encountered, the clinician should be alert for the possibility of a non-deflating urethral catheter. The most common cause of nondeflating catheters is the crystallization of saline within the catheter channels or a fault at the level of the valve mechanism. To remove non-deflating catheters, follow the guidelines [20]:

- Advance the Foley catheter to make sure it is within the bladder.

- If this does not work, slowly aspirate the balloon, when done rapidly, a collapse of the valve mechanism is possible, thus giving into non-deflating catheters.

- If the balloon is still not deflated, then the balloon port can be cut proximal to the inflation valve. This allows for spontaneous drainage of urine.

- If this does not work, a guidewire can be introduced into the inflation channel, and this should pierce and deflate the balloon.

- If the above techniques are unsuccessful, the instillation of $10 \mathrm{cc}$ mineral oil into the inflation port will cause the balloon's dissolution in approximately $15 \mathrm{~min}$.

- When all the above fails, a prompt referral to a urologist is to be made [21, 22].

\section{Complications}

Urethral catheterization is associated with few possible complications such as [23, 24]:

- Bleeding

- Urethral strictures

- Urethral perforations

- Urethral trauma

- Paraphimosis

- Pseudopolyps

- Sepsis: this can arise from infections such as urethritis, pyelonephritis, and cystitis

- Antibiotic resistance

\section{Enhancing Healthcare Outcomes}

As general medical practitioners in private/outpatient settings, the use of urethral catheters is not as frequent as those found inpatient admitted to the hospital. However, the continual and routine catheter management in patients who have been deemed to require an indwelling catheter is usually undertaken by the general practitioners [25].

Regardless of the clinical setting, i.e., inpatient $v s$ outpatient, the appropriate skills, knowledge, and techniques discussed above should be utilized when passing a urethral catheter.

Even though urinary catheterization has become a routine procedure, urinary catheter use should be abstained from when plausible [26]. The use of clean intermittent catheterization is of more significant benefit to the patient over long-term indwelling catheterization. While it is unavoidable that some patients will require the use of long-term indwelling urethral catheters, the routine cleansing of the perineal area and the catheter has not been shown to reduce the incidence of bacteremia.

Irrespective of the type either intermittent long-term, the risk of bacteremia still exists, but more for patients with a long-term indwelling catheter. The routine antibiotics use in patients with urethral catheters is advised against; the patient should only be treated when the infection is symptomatic or polymicrobial in etiology. 
A routine periodic renal scan or cystoscopy may be indicated for patients with an indwelling catheter for over 10 years. These categories of patients are at an increased risk of renal complications and bladder cancer.

Once the decision to place a urethral catheter has been made, conscious efforts focusing on standard hygienic practices should be practiced minimizing any problems associated with catheter management.

The physical and psychological burden of prolonged catheter use, especially for those with indwelling urinary catheters, is significant, and it negatively affects the patient's QoL. For patients with acute urinary retention (AUR) ameliorable to surgery, such should be scheduled faster to reduce numerous potential complications and burdens linked with prolonged catheter use.

A cordial and patient-centered inter-professional relationship among healthcare providers such as general surgeons, urologists, nursing practitioners, and the general physician will bring about the best patient outcome [27].

\section{Important Message}

- Utilize appropriate size catheters during male urethral catheterization: do not use shorted women catheters for males.

- The use of sterile water is preferred over regular saline dues to a possibility of saline crystallization.

- Replace the foreskin in uncircumcised male post-procedure to prevent paraphimosis.

- Document the residual urine volume in retention.

- After two unsuccessful attempts at catheterization, call for help if available or refer to the nearest urgent care or emergency department [5].

\section{References}

1. Hume EH. Medicine in China, old and new. Annals of Medical History. 1930;2:272-80.

2. Nacey J, Delahunt B. The evolution and development of the urinary catheter. Aust N Z J Surg. 1993;63(10):815-19.

3. Feneley RC, Hopley IB, Wells PN. Urinary catheters: history, current status, adverse events and research agenda. J Med Eng Technol. 2015;39(8):459-70.

4. Wyndaele JJ. Intermittent catheterization: which is the optimal technique? Spinal Cord. 2002;40(9):432-37.

5. Shah J. Catheterization. Ann R Coll Surg Engl. 2012;94(1):5-7.

6. Moore KL, Dalley AF II, Agur AM. The bladder \& Urethra. In: Moore KL, eds. Clinically oriented anatomy. 7th ed. Philadelphia: Wolters Kluwer Health/Lippincott Williams \& Wilkins; 2014. p. 362-68.

7. Shermadou ES, Rahman S, Leslie SW. Anatomy, Abdomen and Pelvis, Bladder. StatPearls. 2020.

8. Lanzotti NJ, Tariq MA, Bolla SR. Physiology, Bladder. StatPearls. 2020.

9. Schenkman NS. Male Urethra Anatomy. 2016.

10. Shlamovitz GZ. Urethral Catheterization in Men. 2016.

11. Tyagi P, Tyagi S, Kaufman J, et al. Local drug delivery to bladder using technology innovations. Urol Clin North Am. 2006;33(4):519-30.

12. GuhaSarkar S, Banerjee R. Intravesical drug delivery: Challenges, current status, opportunities and novel strategies. J Control Release. 2010;148(2):147-59. 
13. Essman SC. Contrast cystography. Clin Tech Small Anim Pract. 2005;20(1):46-51.

14. Dane B, Baxter AB, Bernstein MP. Imaging genitourinary trauma. Radiol Clin North Am. 2017;55(2):32135 .

15. Hadfield-Law L. Male catheterization. Accid Emerg Nurs. 2001;9(4):257-63.

16. Gerard LL, Cooper CS, Duethman KS, et al. Effectiveness of lidocaine lubricant for discomfort during pediatric urethral catheterization. J Urol. 2003;170(2 Pt 1):564-67.

17. Thomsen TW, Setnik GS. Videos in clinical medicine. Male urethral catheterization. N Engl J Med. 2006;354(21):e22.

18. Corder CJ, LaGrange CA. Suprapubic Bladder Catheterization. StatPearls. 2020.

19. Hilton P, Stanton SL. Suprapubic catheterisation. Br Med J. 1980;281(6250):1261-263.

20. Shapiro AJ, Soderdahl DW, Stack RS, et al. Managing the nondeflating urethral catheter. J Am Board Fam Pract. 2000;13(2):116-19.

21. Joseph R, Huber M, Leeson B, et al. Ultrasound-guided Placement of a Foley Catheter Using a Hydrophilic Guide Wire. Clin Pract Cases Emerg Med. 2018;2(2):143-46.

22. Willette PA, Coffield S. Current trends in the management of difficult urinary catheterizations. West J Emerg Med. 2012;13(6):472-78.

23. Wyndaele JJ. Complications of intermittent catheterization: their prevention and treatment. Spinal Cord. 2002;40(10):536-41.

24. Davis NF, Quinlan MR, Bhatt NR, et al. Incidence, cost, complications and clinical outcomes of Iatrogenic urethral catheterization injuries: A prospective multi-institutional study. J Urol. 2016;196(5):1473-77.

25. Bhatia N, Daga MK, Garg S, et al. Urinary catheterization in medical wards. J Glob Infect Dis. 2010;2(2):8390 .

26. Meddings J, Rogers MA, Krein SL, et al. Reducing unnecessary urinary catheter use and other strategies to prevent catheter-associated urinary tract infection: an integrative review. BMJ Qual Saf. 2014;23(4):277-89.

27. Ikuerowo SO, Ogunade AA, Ogunlowo TO, et al. The burden of prolonged indwelling catheter after acute urinary retention in Ikeja - Lagos, Nigeria. BMC Urol. 2007;7:16. 